\title{
Psychiatry in Barbados: a personal experience
}

\author{
Ceri Evans
}

As a registrar, I took part in a six-month exchange between the Maudsley Hospital in London and the Psychiatric Hospital in Barbados. My main motivation for taking up this opportunity was a long-standing desire to spend at least part of my psychiatric training in a less well-developed setting. My expectations were that Barbados would provide this kind of experience, with the emphasis being on service delivery in a very different cultural setting to which I was accustomed.

Barbados is the eastern-most Caribbean island, with a population of around 260000 people centred mainly around the capital, Bridgetown. It is one of the more developed Caribbean islands, with a relatively strong currency, and the second highest literacy rate in the world. Although Barbados enjoys its well-founded reputation as an idyllic tourist destination, life for the majority of the population outside of the resorts is far from lavish. Afro-Caribbeans account for $95 \%$ of the population.

\section{Psychiatric services}

Services catering for the mentally ill in Barbados have developed since the opening of The Mental Asylum on a plantation called Jenkinsville in 1893. Although remaining on this original site. the current facility is called the Psychiatric Hospital', which is the largest of the three different psychiatric resources operating in Barbados. The other two services are a 12 bedded unit in Queen Elizabeth Hospital (the main general hospital near Bridgetown), which cares mainly for patients suffering from depression or following deliberate self-harm, and the Community Services, involving both nurse-run psychiatric clinics in the regional 'Polyclinics', and domiciliary visits by district nurses (up to 6000 per year).

The Psychiatric Hospital cares for around 630 in-patients and 30 day-patients at any one time, with the patient gender ratio comprising around 2.5 men for every female patient. Almost half of the in-patients were considered 'long-stay', meaning that discharge would not foreseeably occur. The medical staff consisted of three consultant psychiatrists and five or six junior staff. There were 10 wards including two forensic wards (one medium security, one high security), and a ward for the 'subnormals' (the accepted local term for the learning disabled). The general wards were divided along gender lines, and catered for up to 70 patients at a time, with sleeping arrangements either in dormitory format or in single concrete rooms which were celllike' in appearance (see Fig. 1). Each year, there are over 200 first admissions, and 900 readmissions.

The low staff to patient ratios contributed to medication being the main treatment on offer. with the drug and alcohol service providing the main psychotherapeutic input. In addition, the lack of alternative facilities lead to some chronically medically ill patients being kept at the Psychiatric Hospital. This lack of other resources

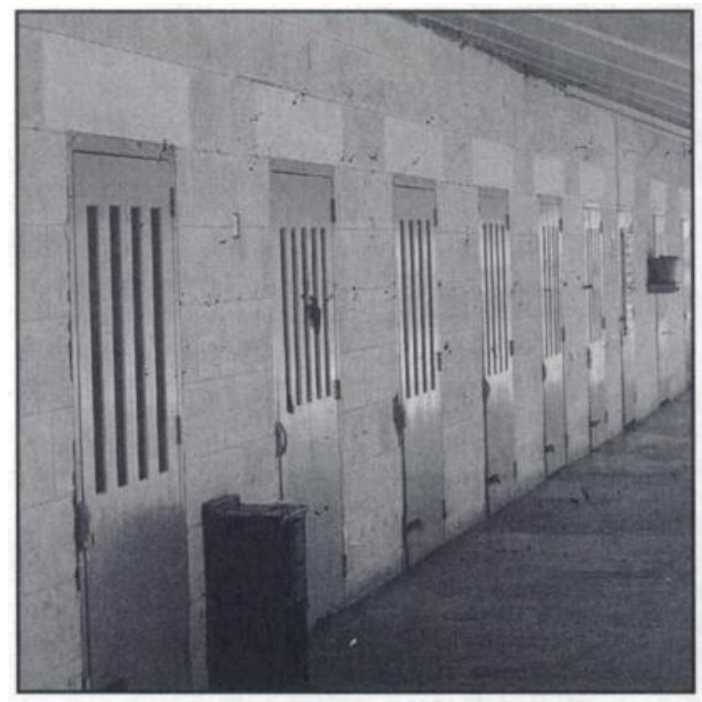

Fig. 1. Series of patients individual rooms on ' $G$ ' ward. 
also meant that the question of not admitting a patient because of a lack of beds never arose; a bed was always found somewhere for an acute admission.

The socio-political context impacted upon the delivery of psychiatric care in a variety of ways. The public perception of the Psychiatric Hospital was largely that of a custodial institution, a view reinforced by the high profile afforded mentally disordered offenders by the local media. Moreover, the Barbados Police Force had a low threshold for dropping off 'patients' for admission when the symptoms were "causing a disturbance', particularly if they appeared to be intoxicated, and almost certainly if they had a history of psychiatric admission. The local Mental Health Act was reviewed in 1987, with the patients having reduced rights as judged from the UK perspective. For example, a voluntary admission required the patient to give 24-hours notice of their intended departure, and there has only been one appeal (unsuccessful) against compulsory detention in recent years. There were also more positive social aspects influencing the delivery of psychiatric care: the stigma associated with mental illness in Barbados meant that prospective patients were often cared for by impressive extendedfamily structures well after admission was indicated.

\section{Clinical and academic experience}

My clinical responsibilities during the registrar exchange included duties with the Liaison Psychiatry service at Queen Elizabeth Hospital. the Child Guidance Clinic, and the Drug and Alcohol Rehabilitation Team, in addition to participation in a one in five on-call rota.

The Liaison Service was in its relative infancy. and as such dealt mostly with standard referrals involving florid psychiatric presentations of organic disorders, deliberate self-harm, and depressive illnesses. It is likely that more sophisticated liaison work involving preventative or rehabilitative care within a multi-disciplinary setting will develop as the service matures.

The Child Guidance Clinic was a weekly outpatient clinic in which around three new referrals and 10 'returns' were assessed. The appointment system of giving the day of the clinic only, combined with the 'first come, first served' basis for seeing patients meant that most of the days' patients, along with their mothers and siblings, were there when I first arrived: fourhour waiting times were commonplace. The most frequent referral was for conduct disorder, which was invariably related to difficult social circumstances. New cases of hyperactivity, childhood depression, organic delusional disorder following head injury, and mental retardation diagnosed at age 12, all presented to the Child Guidance Clinic during my attachment.

The Drug and Alcohol Rehabilitation Team functioned with a six-week residential programme involving daily groups and relapse prevention work as its centrepiece, with little out-patient or brief intervention work carried out. The drugs of abuse in Barbados are more or less restricted to just three: alcohol (in the form of rum), ganja (marijuana) and crack cocaine. Bajans apparently do not like 'breaking' their skin (hence deliberate self-harm rarely occurs by cutting) and therefore injections are avoided, with opiate abuse minimal in Barbados. Use of ganja is seen as a serious and relatively deviant activity in Barbados, which runs contrary to the West Indian stereotype held in the UK. There is a firm local belief in the causation of both acute and chronic psychotic syndromes by ganja use.

The clinical work generated some memorable personal moments, including: two separate patients appearing perplexed and anxious as to why I wanted to enlist the help of a priest, when in fact I had asked them in my kiwi accent if they were depressed (de Priest!); having a mother and her daughter use my opened books as umbrellas to shelter from a tropical downpour inside my office in their desperation to continue their appointment; and seeing the look of astonishment on an elderly Bajan woman's face when a White doctor entered her rural and remote chattel house, chickens and all, on an unannounced domicillary visit.

I also undertook a research project in forensic psychiatry, which gave me the time-consuming but patience-generating experience of liaising between different Police and Government departments, who were, on the whole, very helpful. There were advantages to doing research in Barbados. The medical records department at the Psychiatric Hospital is legendary in its efficiency, the island geography helped ensure a relatively captive population for epidemiological work, and the existence of only one of each major institution or facility such as the prison, the Psychiatric Hospital and the general hospital made chasing down notes a more amenable prospect.

\section{Merits of the exchange}

In my view there were several worthwhile benefits associated with a training experience of this kind. First, my understanding of the degree of cultural dislocation which must be experienced by West Indians living in the UK was irreversibly enhanced by having first-hand exposure to an undiluted version of one West Indian culture (I was constantly told that each country in the West Indies jealously protected 
and emphasised what they perceived as significant cultural differences between the countries). I would not claim to have expert cultural knowledge as a result of the exchange, but I do think that my awareness of the relevance of cultural issues to individual cases has been fine-tuned. Second, my understanding of the difficulties of psychiatric service delivery in the context of truly scarce resources, was improved. This also helped both sharpen various views about patients' rights, resource management, organisational structures, and social discrepancies, and to (on reflection, and predictably) soften my initial paternalistic attitudes towards service delivery supported by comparatively few resources. Without becoming nihilistic in my approach. I certainly came to accept more fully the limitations imposed by serious financial constraints, and, just as powerfully, the inherent resistance to significant institutional change. Third, I had the unusual experience of being, in a very clear way, an outsider in an institution, with my White skin setting me apart from all other staff and patients. Being followed (sometimes completely surrounded by patients as I walked along), touched, jabbed and being called 'Whitey' (and variations thereof) was certainly a thought provoking experience. Fourth, the opportunity to do research of an epidemiological kind reinforced the training merits of the exchange. Last, I now have a better sense of life in psychiatry outside of such a well-resourced institution, which will no doubt give me an additional perspective with which to confront and evaluate my ongoing training experiences. I regularly reflect on the exchange, and I anticipate that in future years, some of the views and opinions I will put forward in psychiatric settings might be informed by my Barbados experience.

\section{Acknowledgements}

I thank Dr Ian Kerr for his helpful suggestions on early drafts of this article, and Dr Ermine Belle, Dr George Mahy and Dr Sharon Harvey (all Barbados) for their help and support during this exchange.

Ceri Evans, Registrar in Psychiatry, Maudsley Hospital, Denmark Hill, London SE5 8AZ

\section{Antisocial Personality Disorder: An Epidemiological Perspective}

\section{by Paul Moran}

In 1980, the controversial diagnostic category of 'antisocial personality disorder' was introduced in psychiatry. This book provides a comprehensive review of antisocial personality disorder from an epidemiological point of view. It opens with a discussion of the central problems associated with assessing and classifying abnormal personality and then focuses more specifically on antisocial personality disorder with chapters on: distribution, natural history, early risk factors, associated conditions, burden and needs assessment. It will be a valuable source of reference for all who are interested in the disorder, whether from a clinical, management or research perspective.

January 1999, s12.50, 144pp, ISBN 1901242242

Avallable from Book Sales, Royal College of Psychiatrists, 17 Belgrave Square, London SWLX 8PG Tel +44 (0) 1712352351 (extension 140), Fux +44 (0) 1712451231

http://www.rcpsych.ac.uk 